\title{
Investigation of the impact of simulated solar radiation on the micro- and nanoscale morphology and mechanical properties of a sheet moulded composite surface
}

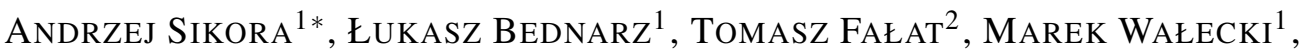 \\ MARIA ADAMOWSKA ${ }^{1}$ \\ ${ }^{1}$ Electrotechnical Institute, Division of Electrotechnology and Materials Science, \\ M. Skłodowskiej-Curie 55/61, 50-369 Wrocław \\ ${ }^{2}$ Wrocław University of Technology, Faculty of Microsystem Electronics and Photonics, \\ Janiszewskiego 11/17, 50-372 Wrocław
}

\begin{abstract}
In this paper we present the results of investigation of micro- and nanoscale degradation of a sheet moulded composite exposed to simulated solar radiation. Utilization of high resolution methods such as atomic force microscopy, optical profilometry and microcomputer tomography allowed us to provide the evidence of significant deterioration of the surface as well as the material few microns in depth. Additionally, the typically used macroscopic investigations, such as wettability and flexural strength, were performed to observe the impact of weathering process. It was also shown that high resolution techniques provide superior sensitivity of the material degradation detection. The particular effectiveness of the applied approach was related to the structure of investigated material, as due to its degradation, a number of voids appeared, causing a significant roughness increase. In addition, the impact of light radiation could be compared to other environmental conditions maintained in the climatic chamber. It should be underlined, that according to our knowledge, such a study has not been performed so far.
\end{abstract}

Keywords: weathering; material decomposition; sheet moulded composite; atomic force microscopy; microcomputer tomography

(c) Wroclaw University of Technology.

\section{Introduction}

Recent progress in material science is possible due to access to advanced and complex diagnostic tools and methods. Enabling a new measurement methodologies may allow to improve the investigation procedures in terms of accuracy, speed and costs. One of the most common tests of materials, is verification of the influence of environmental conditions. One can list a number of procedures defined in the standards in order to observe the deterioration caused by the simulated solar light (i.e. PN-EN 60068-2-5:2011) as well as changing temperature and humidity (i.e. PN-EN 60068-2-38:2010). After the sample is exposed to certain conditions, the verification of the mechanical properties changes, such as tensile properties

*E-mail: sikora@iel.wroc.pl
(PN-EN ISO 527-2:2012), Charpy impact strength (PN-EN ISO 179-1:2010) or others may be performed. It should be underlined that the number of samples required for such tests, and the energy necessary to run the environmental chamber (even for few hundreds of hours) may generate a significant cost. Therefore, a development of a new test solutions is desired in order to reduce the process expenses as well as to reduce the time of a new product tests and release. In particular, high sensitivity and high resolution measurement techniques, such as atomic force microscopy [1], optical profilometry $[2,3]$ and $\mathrm{X}$-ray microcomputer tomography (CT) $[4,5]$ can be successfully used for determination of the material deterioration. Such methods can provide the information about the decomposition of the material after applying of smaller doses of certain medium than in case of standard tests. Also, in case of nanomaterials, such tools are 
useful in terms of observation of specific submicron components behavior after specific exposition is applied.

It should be emphasized that the diversity of atomic force microscopy techniques (AFM) allows one to perform imaging of mechanical, magnetic, electrical, thermal and optical properties of the surface [1]. A number of observations of the morphology change due to the light/temperature or electrical field [6-10] have been performed. One has to be aware that significant variations of certain properties due to the local inhomogeneities of material can become an obstacle during the measurements while the abovementioned methods are utilized. However, the issue of precise positioning was efficiently solved, as so called nanomarkers allow us to perform various measurements in the same area of interest, providing highly reliable experimental outcome [10-13].

The sheet moulded composite is one of very popular materials due to its remarkable mechanical properties and easy processing method. It is often utilized in electrical devices such as housing boxes for cable connectors, switches, fuses and many others. In outdoor applications, the sun radiation impact test is essential in terms of user safety. Therefore, in this paper, we present the results of advanced measurement techniques allowing to observe deterioration of the samples. The applied tests revealed the early stages of material degradation, which resulted in further dynamic deterioration of the components.

\section{The sample and the test proce- dure}

A SMC (sheet moulded composite) sample was prepared using standard process, then it was cut into pieces so as to meet the PN-EN ISO 179-1:2010 standard requirements. The samples (SMC C) were exposed to simulated solar radiation according to PN-EN ISO 4892-2:2009 standard. The test conditions i.e.: spraying period: 5 minutes every 30 minutes; spectral radiation intensity: $0.51 \mathrm{~W} / \mathrm{m}^{2} \cdot \mathrm{nm}(340 \mathrm{~nm})$; total radiation energy: $918 \mathrm{~kJ} / \mathrm{m}^{2}(340 \mathrm{~nm})$; radiation spectrum:
$290 \mathrm{~nm} \leqslant \lambda \leqslant 800 \mathrm{~nm}$; black panel temperature: $63.8{ }^{\circ} \mathrm{C}$, were applied for 500 hours. Additionally, another sample (SMC B) was placed in the test chamber without access of the light. For this sample, only the impact of temperature and humidity was investigated to enable a comparison of the effect of both sets of conditions. The reference sample (SMC A) allowed us to compare investigated properties of the material in order to obtain quantitative information about the influence of the applied conditions. The deterioration of the material was verified using following diagnostic methods and instruments: optical profilometry: ContourGT from Bruker, X-ray microcomputer tomography: Nanome|x 180 from General Electric, and atomic force microscopy: Innova from Bruker (former Veeco).

As the optical profilometry does not require any particular preparation procedure, the samples were scanned as removed from the chamber. AFM measurements were performed in following modes: intermittent contact (Tapping Mode) [14], NanoSwing $[15,16]$ and force spectroscopy [17, 18]. The quantitative $3 \mathrm{D}$ reconstruction of the surface was provided by the AFM (Fig. 1 to Fig. 3) and optical profilometry (Fig. 4), which enabled the roughness determination according to specific standards [19]. The data was processed using SPIP software from Image Metrology [20]. In addition, the surface topography data acquired using AFM technique was processed using the Sobel transform, which is an edge detection filter [21]. This particular feature allows one to observe the presence of fine morphological features while the typical color scale in standard topography image does not provide sufficient dynamics to make them clearly visible. It was successfully used in a number of papers [22-24]. It should be stressed, that both the abovementioned methods may give various values, as different physical phenomena and principles of surface reconstruction are utilized and various kinds of artefacts may have an impact on the measurements. Therefore, one must compare the data acquired with the specific techniques. The measurements allowed us to obtain the information about morphological and mechanical properties 

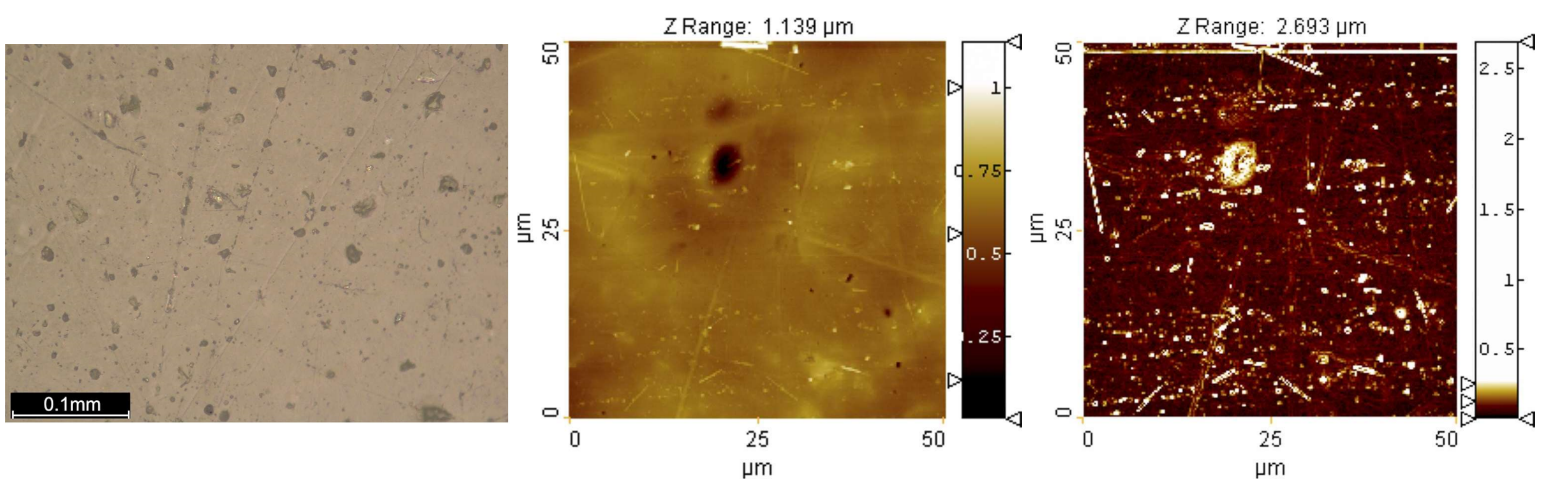

(a)
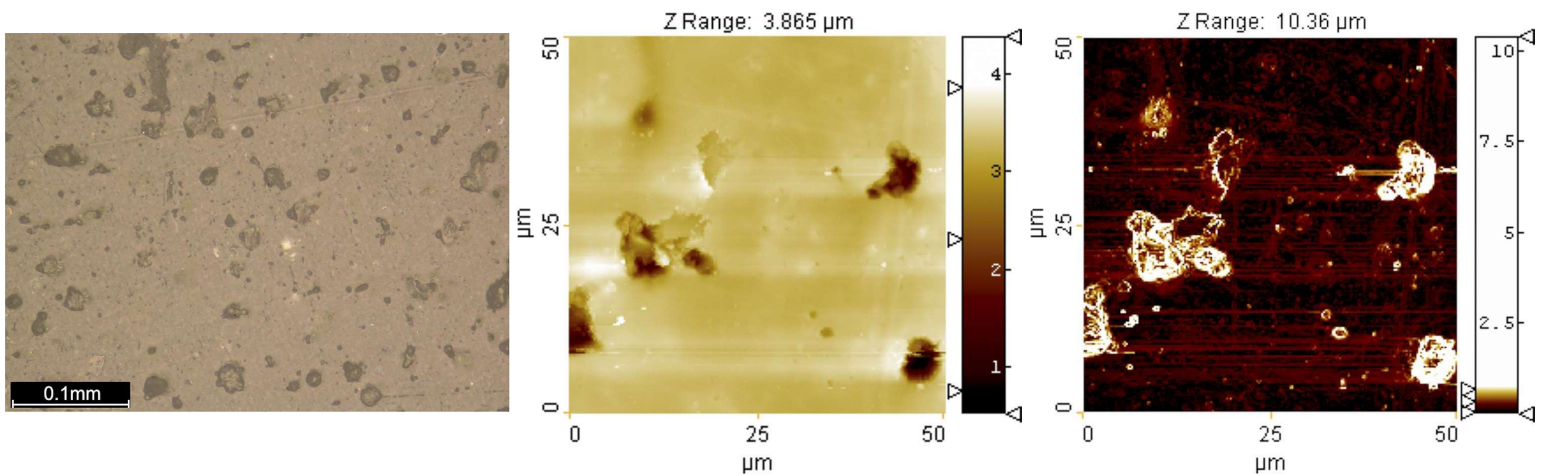

(b)
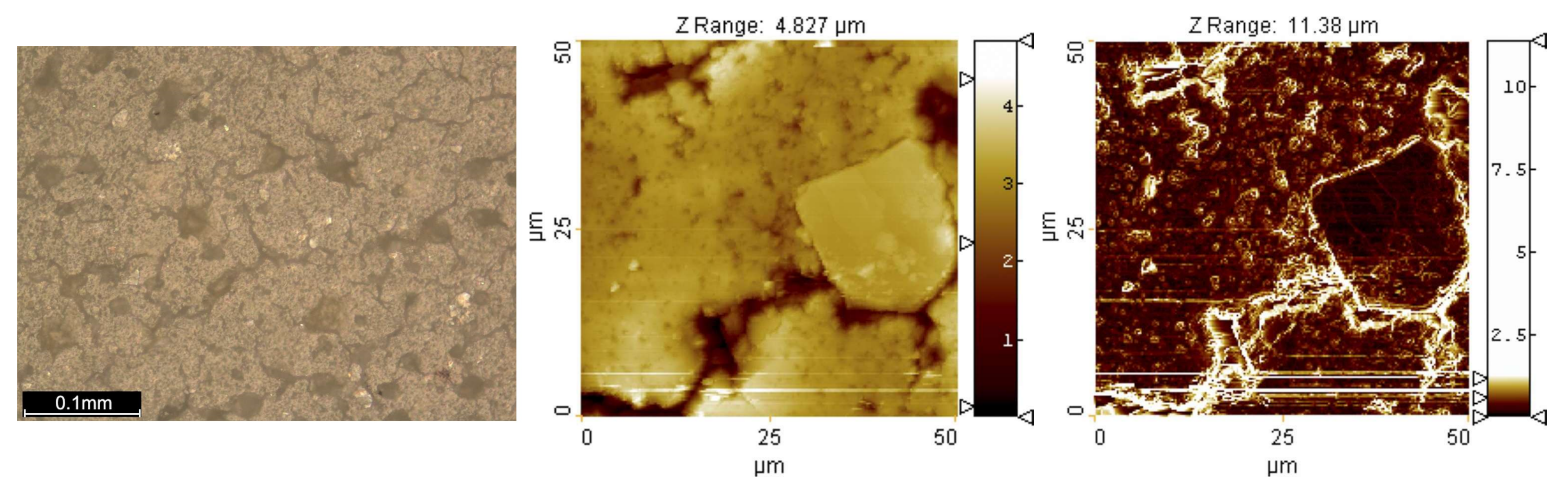

(c)

Fig. 1. Optical microscope images (left hand side), AFM topography images (middle) and the Sobel transform of AFM topography (right hand side), of the SMC A sample (top), SMC B (middle) and SMC C (bottom).

of the material at various stages of its decomposition. In order to avoid the influence of the inhomogeneity of the surface and to provide representative data, the measurements were performed in few spots on each sample. Average values along with standard deviation were calculated. CT investigations required preparation relatively small samples (approximately $3 \mathrm{~mm} \times 3 \mathrm{~mm} \times 3 \mathrm{~mm}$ ), as maximum spatial resolution was assumed (300 nm per voxel). Moreover, in order to compare the obtained data to the standard defined approach, the flexural strength and contact angle were also determined. For the determination of the flexural strength, the tests were performed according to the standard PN-EN ISO 14125:2001. The samples were prepared by cutting into desired size. 
Before the test, the samples were conditioned for 24 hours in the temperature of $24{ }^{\circ} \mathrm{C}$ and the relative humidity of $50 \%$ conditions. The commercial setup 55R6025 from Instron was used with a standard speed of $2 \mathrm{~mm} / \mathrm{min}$. The wettability was investigated using a home-made setup with a microcontroller-based dosing system and digital microscope. Acquired images were processed using a specialized software. For both techniques a series of measurements have been performed in order to provide statistically verified outcome.

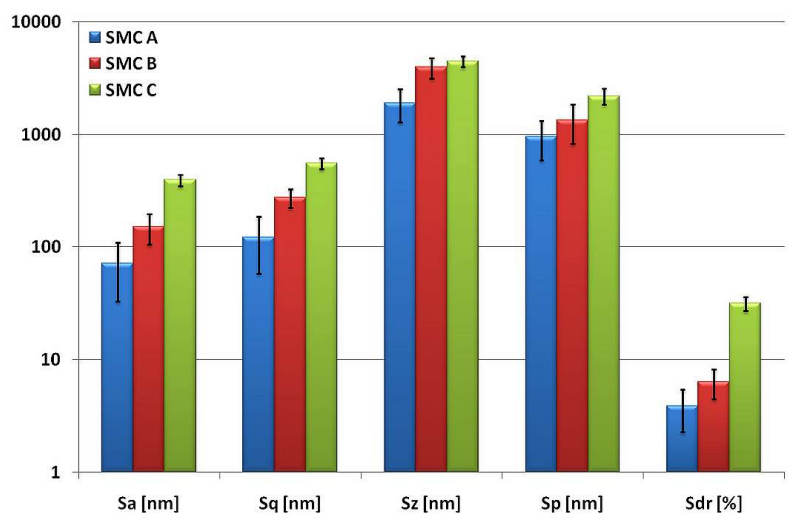

Fig. 2. Roughness parameters of investigated samples determined using AFM topography data.

\section{Measurements results}

Although optical microscopy allowed us to observe the major differences between samples SMC A, SMC B and SMC C during decomposition of the material causing surface degradation (Fig. 1), the quantitative data were needed for objective samples evaluation. The following surface roughness parameters [25]: average roughness (Sa), root mean square roughness $(\mathrm{Sq})$, peak-peak value $(\mathrm{Sz})$, maximum peak height (Sp) and surface area ratio (Sdr) revealed a significant deterioration caused by the presence of both high temperature and humidity as well as the simulated solar radiation (Fig. 2 and Fig. 4). The pores parameters: length, perimeter and $\mathrm{Z}$ maximum were also calculated, providing clear indication of the morphology degradation. The differences of certain factors values reached even an order of magnitude. Also the data acquired with optical profilometer (Fig. 4) showed a significant increase of the abovementioned parameters, even up to almost two orders of magnitude. Another source of information about the morphological changes of the material was the microcomputer tomography. The example images showing the voids density difference between SMC A and SMC C at a depth of $1.5 \mu \mathrm{m}$ from the surface are shown in Fig. 5. Quantitative analysis allowed us to compare the voids volume and breadth of the voids as well as the surface (calculated using 2D images) of the material within the range of depth from $0 \mu \mathrm{m}$ to $15 \mu \mathrm{m}$. The increase of the void area (lateral) and decrease of the material volume along with the increase of the breadth of the void spaces can be noticed (Fig. 5). Basing on the obtained results, one can conclude about the degradation of the material also at certain depth, below the observed surface. It should be underlined that the sheet moulded composite may contain void spaces inside its volume, as the ones created during the fabrication process. These voids are, however, protected against water penetration by the outer layer of the material. When this barrier is damaged by environmental conditions, rapid degradation starts, as the material can be easily penetrated by the media increasing the intensity of this process. The increase of the roughness has a significant impact on the wettability (Fig. 6), which may induce more significant contribution of water in the degradation of the material.

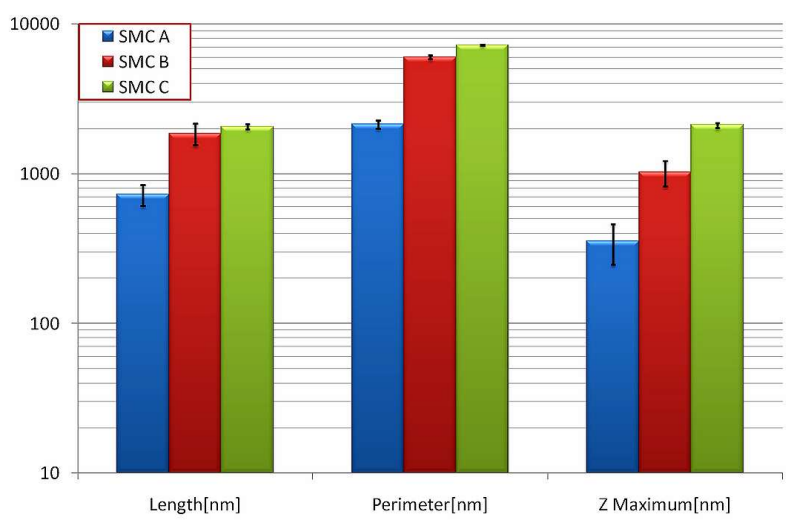

Fig. 3. Pores parameters of investigated samples determined using AFM topography data.

As a consequence of the deterioration of the surface, the decrease of mechanical properties is 

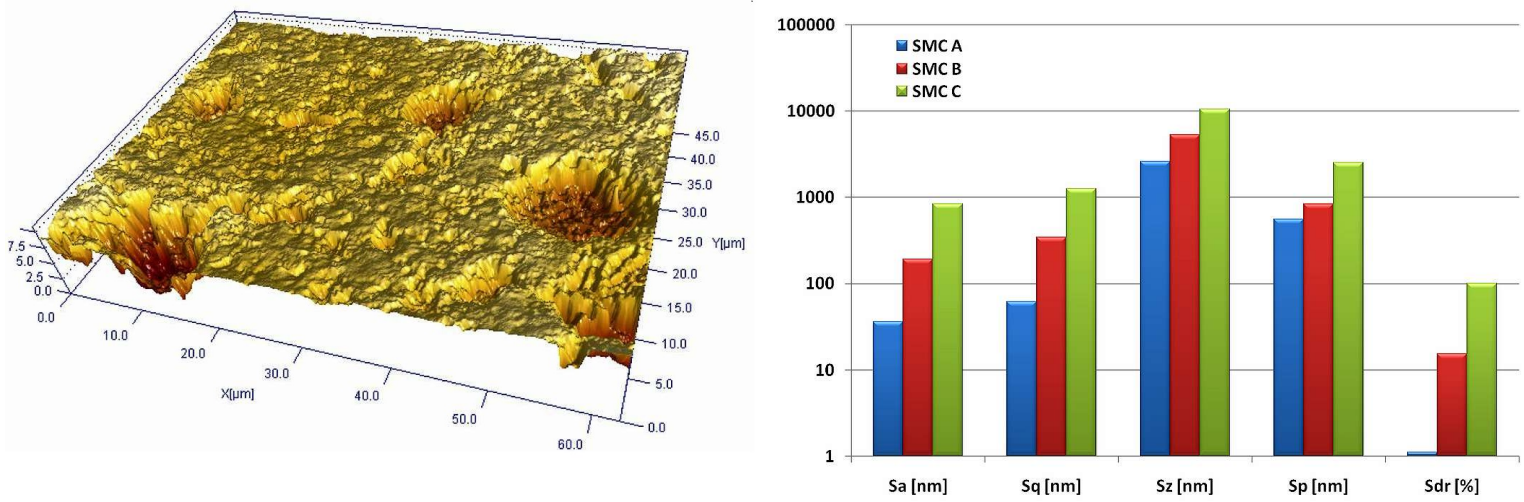

Fig. 4. 3D image of the SMC C surface acquired with optical profilometer (left hand side) and roughness parameters of investigated samples determined using optical profilometry data (right hand side).
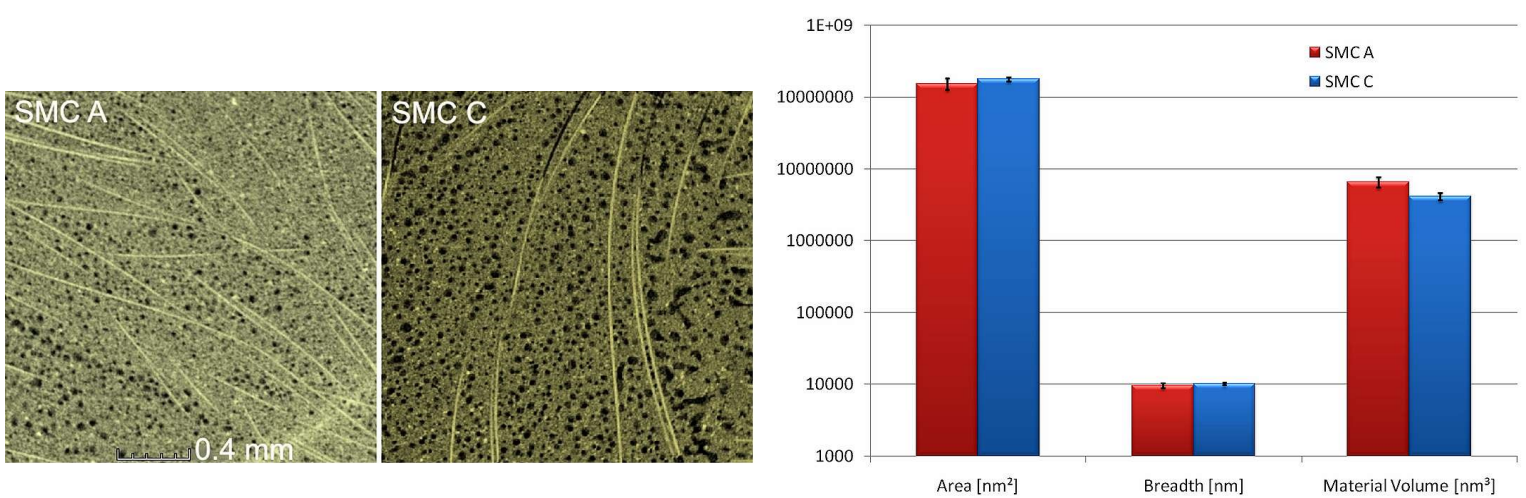

Fig. 5. Microcomputer tomography data acquired at the depth of $1.5 \mu \mathrm{m}$ from the surface of the sample (left hand side) and quantitative comparison of the voids planar area, and the void breadth as well as the material volume (right hand side).
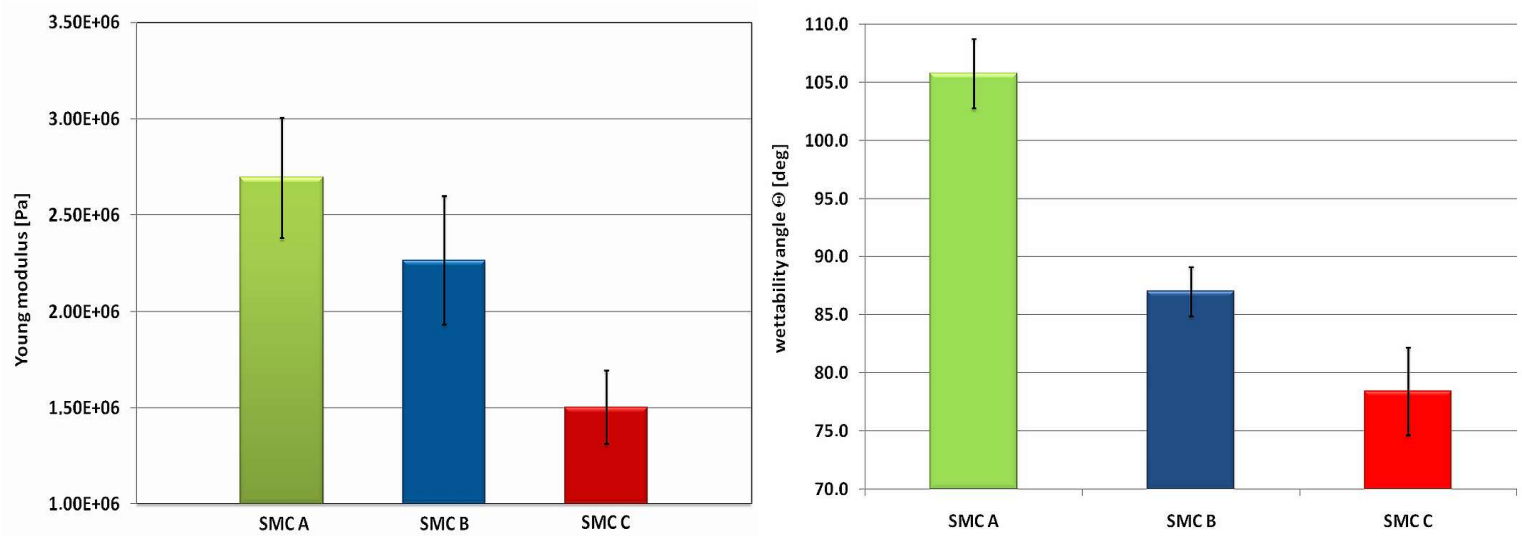

Fig. 6. The Young modulus measured using force spectroscopy (left hand side) and wettability of the surface of investigated samples (right hand side). 

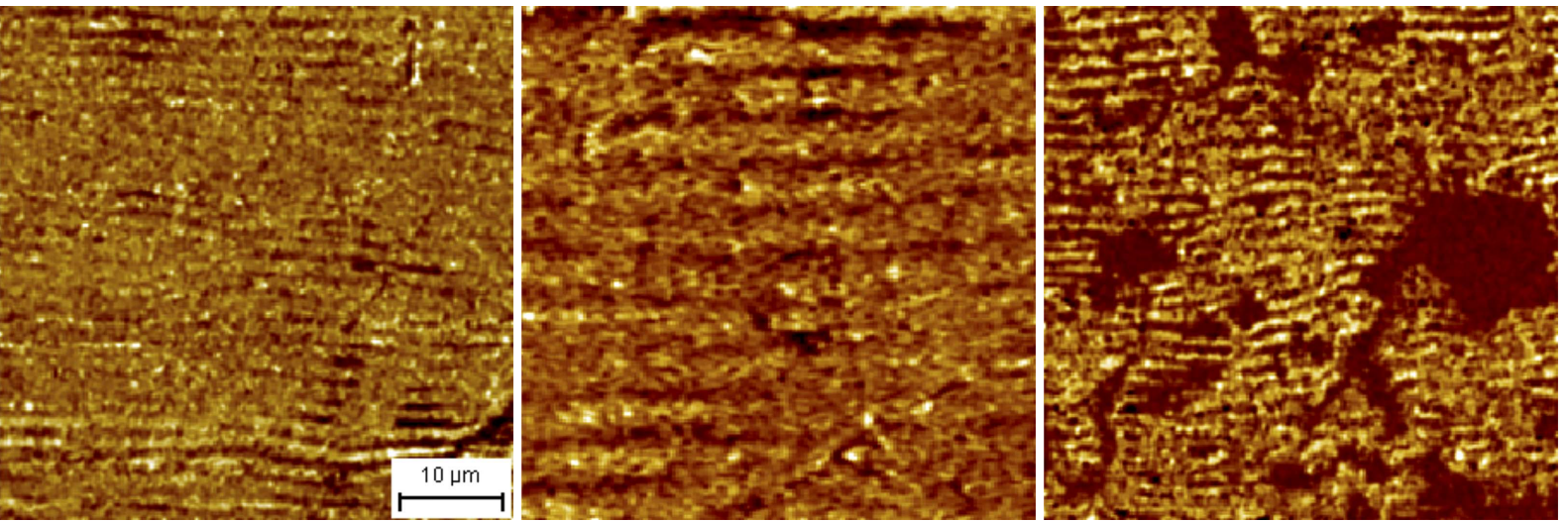

Fig. 7. Energy dissipation for the surface deformation maps. The data was acquired using NanoSwing technique. From left to right: SMC A, SMC B, SMC C. Scan area: $50 \mu \mathrm{m} \times 50 \mu \mathrm{m}$.

expected. The Young modulus was determined using the force spectroscopy technique. A significant decrease of this parameter can be easily noted (Fig. 6). In addition, the surface was mapped using NanoSwing technique as a method enabling the observation of inhomogeneities of stiffness, energy dissipation and adhesion. In order to calculate the above mentioned parameters, DMT model [26] was utilized in the NanoSwing software [16]. As the comparison of the properties was the main goal, the arbitral units were sufficient in presented analysis. Fig. 7 shows the increase in the energy dissipation related to the material density decrease due to the presence of voids under the thin layer of the material. As the material loses its density, it can absorb more energy from the tapping probe. The graph showing the distribution of the energy dissipation is presented in Fig. 8. The peak values for SMC A, SMC B and SMC C are: 0.104, 2.293 and 3.845, respectively.

In addition, the nanoindentation measurements were performed using the AFM system [27]. The diamond-coated probes were utilized in order to avoid the tip wear [28]. The acquired data was processed using SPIP software. The obtained results show an increase of the indentation depth (hf). The projected area values also indicate the deterioration of the material, however, significant changes can be noticed for the sample SMC C (Fig. 9).

Obtained results are coherent with the mechanical tests (PN-EN ISO 14125:2001) which revealed $3 \%$ flexural strength decrease.

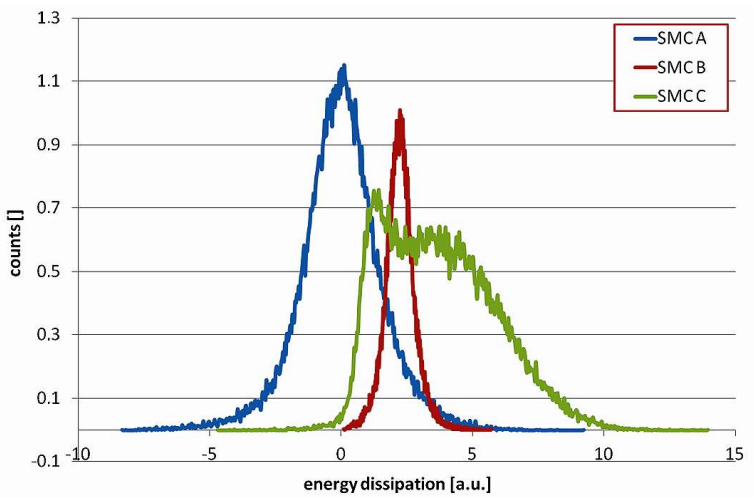

Fig. 8. The distribution of energy dissipation for surface deformation. The data was acquired with NanoSwing technique.

Therefore, the better insight into the deterioration process could be obtained using the novel techniques. The high resolution methods revealed significantly better sensitivity (even by almost two orders of magnitude) than the classical approach. Table 1 summarizes the ratios of certain factors obtained for each measurement technique in order to emphasize the advantages of the new measurement methods. Such an advantage is related to detection of surface parameters, where the solar radiation has the most severe impact (Fig. 10). Therefore, the presented methods can be used for performing faster and cheaper test procedures of materials as smaller samples are required than for standard tests. Also the time of exposition to certain media can be reduced. Such an approach may be particularly effective during 
Table 1. The ratios of selected factors obtained for presented measurement techniques showing the sensitivity of detection of SMC surface deterioration.

\begin{tabular}{cccc}
\hline & Parameter & \multicolumn{2}{c}{ B/A ratio C/A ratio } \\
\hline \hline & $\mathrm{S}_{\mathrm{a}}$ & 2.11 & 5.50 \\
& $\mathrm{~S}_{\mathrm{q}}$ & 2.24 & 4.53 \\
& $\mathrm{~S}_{\mathrm{Z}}$ & 2.08 & 2.35 \\
Atomic force microscopy & $\mathrm{S}_{\mathrm{p}}$ & 1.41 & 2.31 \\
& $\mathrm{~S}_{\mathrm{dr}}$ & 1.64 & 8.19 \\
& Length & 2.55 & 2.83 \\
& Perimeter & 2.81 & 3.39 \\
& $\mathrm{Z}$ max & 2.89 & 5.98 \\
& Young module & 0.84 & 0.56 \\
& Energy dissipation & 22.05 & 36.97 \\
\hline & $\mathrm{S}_{\mathrm{a}}$ & 5.23 & 23.20 \\
& $\mathrm{~S}_{\mathrm{q}}$ & 5.52 & 20.37 \\
& $\mathrm{~S}_{\mathrm{z}}$ & 2.07 & 4.03 \\
Optical profilometry & $\mathrm{S}_{\mathrm{p}}$ & 1.49 & 4.50 \\
& $\mathrm{~S}_{\mathrm{dr}}$ & 13.80 & 90.63 \\
\hline \multirow{4}{*}{ Microcomputer Tomography } & Area & - & 1.16 \\
& Breadth & - & 1.07 \\
& Material Volume & - & 0.63 \\
\hline Wettability & & 0.91 & 0.73 \\
\hline Impact strength & & - & 0.97 \\
\hline
\end{tabular}

the preliminary tests, where the number of the samples is large; the "small dimension-large quantity" approach may be cost-effective [29]. It should be noted that the obtained data allowed us to recognize the difference between the impact of high temperature and humidity only, and combined high temperature, humidity and light radiation. The increase of the material deterioration caused by the radiation is, in general, easy to observe in a form of numerous submicron fractures, leading to rapid progress of submilimeter decomposition of the surface.

\section{Summary and outlook}

In this paper we presented the possibility of using high resolution based techniques in the investigation of deterioration of a sheet moulded composite caused by the simulated solar radiation. Using of the optical profilometry, atomic force microscopy and microcomputer tomography was demonstrated to prove their superior sensitivity for quantifying and comparing the morphological and mechanical properties of the surface in terms of the impact of environmental conditions. The changes in these factors reached even few orders of magnitude, while in case of standard techniques, such as the flexural strength, only few percent decrease was revealed. Therefore, basing on the results obtained by high resolution methods, one can easily distinguish the degradation level of the samples, and use it for further analysis. It should be underlined that recent development of the methods presented in this paper, allows us to perform the calibration procedure in terms of providing the traceability, which is essential for each test method. Additionally, a unique NanoSwing mapping technique was also presented in terms of providing useful information concerning the mechanical condition of a material. Further research will enable the development of certain models allowing us to correlate the standard-defined approaches with more 

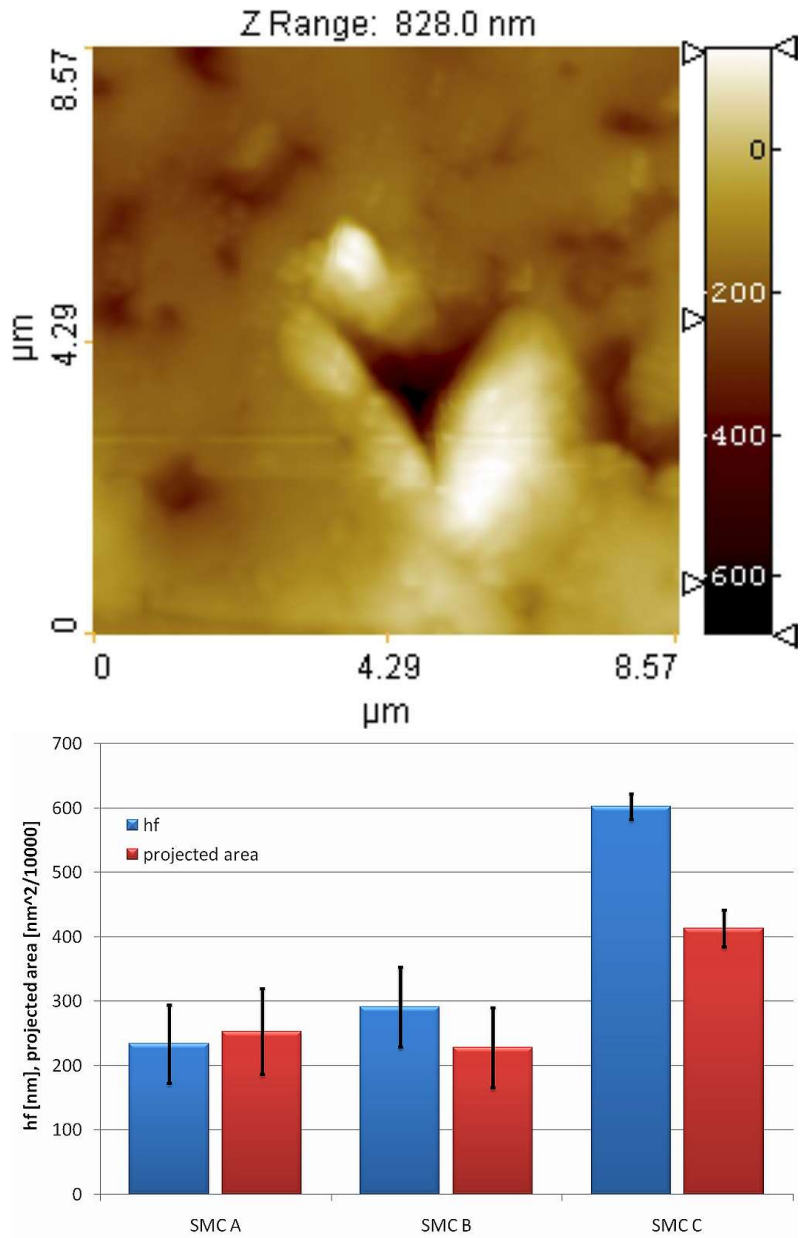

Fig. 9. An example of the nanoindentation trace and a graph showing the changes of indentation depth and projected area of the investigated samples.

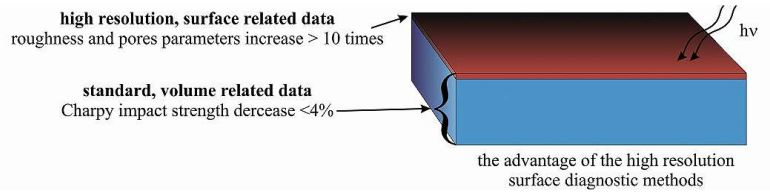

Fig. 10. The advantage of the measurement of the degradation of the surface instead of whole volume of the material.

advanced and complex ones. Such an effort may provide standardized investigation methods allowing us to perform cheaper and faster tests, as smaller samples may be exposed to certain medium for shorter time periods.

\section{Acknowledgements}

This work was performed within the statute funds of the Electro-Technical Institute.

\section{References}

[1] Loos J., Adv. Mat., 17 (15) (2005), 1821

[2] Malinauskas M., Žukauskas A., BELAZARAS K., TIKUiŠIS K., PURlys V., Gadonas R., PISKarskas A., Eur. Phys. J.-Appl. Phys., 58 (2012), 20501.

[3] Salas O., Kearns K., Carrera S., Moore J.J., Surf. Coat. Tech., 172 (2 - 3) (2003), 117.

[4] Soltani P., Johari M.S., Zarrebini M., Powder Technol., 254 (2014), 44.

[5] FaŁat T., Matkowski P., PŁatek B., Zandén C., FELBA J., YE L.-L., LIU J., EMPC 2013, 2013 (2013), 1.

[6] Suresh B., Maruthamuthu S., Khare A., Palanisamy N., MURalidharan V.S., RagUNATHAN R., KANNAN M., PANDIYARAJ K.N., $J$. Polym. Res., 18 (6) (2011), 2175.

[7] Nowicki M., RichteR A., Wolf B., KACZMAREK H., Polymer, 44 (21) (2003), 6599.

[8] Robertson C., Wertheimer M., Fournier D., LAMARre L., IEEE T. Dielec. El. In., 3 (2) (1996), 283.

[9] Minoshima K., Oie Y., Komai K., ISIJ Int., 43 (4) (2003), 579.

[10] Wu J., Zhao M., Nguyen T., Gu X., Mater. Res. Soc. Symp. Proc., 1056 (2008), 227.

[11] Proff C., Abolhassan S., Dadras M.M. Lemaignan C., J. Nucl. Mater., 404 (2010), 97.

[12] Sikora A., Meas. Sci. Technol., 25 (2014), 055401.

[13] SiKorA A., Optica Appl., 43 (1) (2013), 163.

[14] Garcia R., Perez R., Surf. Sci. Rep., 47 (2002), 197.

[15] Sikora A., Bednarz Ł., Przeglad Elektrotechniczny, 11a/2010 (2010), 207.

[16] Sikora A., Bednarz Ł., Cent. Eur. J. Phys., 9 (2) (2011), 372.

[17] Butt H.-J., Cappella B., Kappl M., Surf. Sci. Rep., $59(1-6)(2005), 1$.

[18] SiKora A., Iwan A., High Perform. Polym., 24 (3) (2012), 218

[19] STANDARD ISO/DIS 25178-2 ASME B46.1.

[20] http://www. imagemet. com, accessed 25.11.2015.

[21] VinCENT O.R.F.O, InSITE 2009, 2009 (2009), 97

[22] Iwan A., Boharewicz B., TAzBir I., Sikora A., SChaB-BAlcerZaK E., Grucela-ZajaC M., SKoRKA Ł., Synthetic Met., 189 (2014), 183.

[23] IWAN A., Boharewicz B., PARAFINIUK K., Tazbir I., Gorecki L., SiKora A., FilapeK M., SCHAB-BALCERZAK E., Synthetic Met., 195 (2014), 341.

[24] ChuchmatA A., Palewicz M., Sikora A., IwAN A., Synthetic Met., 169 (2013), 33.

[25] http://www.imagemet.com/WebHelp6/Default. htm\#RoughnessParameters/Roughness_ Parameters.htm, accessed 25.11.2015. 
[26] Derjaguin B. V., Muller V. M., Toporov Y.U.P., J. Colloid Interf. Sci., 53 (1975), 314.

[27] Ekwińska M., RYMuZa Z., Microelectron. Eng., 87 (2010), 1404.

[28] http://www. brukerafmprobes.com/ p-3249-ddesp-10. aspx, accessed 25.11.2015.
[29] Ozimek M., Sikora A., Wilczyński W., Przeglad Elektrotechniczny, 89 (8) (2013), 208.

Received 2016-01-18

Accepted 2016-06-28 